\title{
Bychkov-Rashba Effect and $g$-Factor Tuning in Modulation Doped SiGe Quantum Wells
}

\author{
H. Malissa ${ }^{a}$, W. Jantsch ${ }^{a}$, M. Mühlberger ${ }^{a}$, F. SchäfFleR ${ }^{a}$, \\ Z. Wilamowski ${ }^{b}$, M. Draxler ${ }^{c}$ and P. Bauer ${ }^{c}$ \\ ${ }^{a}$ Institut für Halbleiter- und Festkörperphysik, Johannes Kepler Universität \\ 4040 Linz, Austria \\ ${ }^{b}$ Institute of Physics, Polish Academy of Sciences \\ al. Lotników 32/46, 02-668 Warsaw, Poland \\ ${ }^{c}$ Institut für Experimentalphysik, Johannes Kepler Universität \\ 4040 Linz, Austria
}

\begin{abstract}
We investigate the spin resonance of electrons in one-sided modulation doped $\left.\mathrm{Si}_{1-x} \mathrm{Ge}_{x}(x=0-10 \%)\right)$ quantum wells defined by $\mathrm{Si}_{0.75} \mathrm{Ge}_{0.25}$ barriers. In such structures, the Bychkov-Rashba effect induces an effective magnetic field in the quantum well layer which causes anisotropy of both the $g$-factor and the spin coherence time. Evaluation of the Rashba coefficient as a function of $x$ yields a monotonic increase. For $x=5 \%$ the shift in the resonance field exceeds the ESR linewidth already, demonstrating the possibility to use this effect for $g$-factor tuning to select individual spins in an ensemble.
\end{abstract}

PACS numbers: 73.21.Fg, 85.75.-d

\section{Introduction}

Among the criteria for spintronics and quantum computing are (i) the possibility to manipulate individually addressable spins in a large ensemble and (ii) a spin coherence time that exceeds the manipulation time by orders of magnitude [1]. Low dimensional structures built from $\mathrm{Si}_{1-x} \mathrm{Ge}_{x}$ appear as possible candidates for such tasks. It has been shown that the spin coherence time in Si quantum wells defined by SiGe barriers is of the order of $1-3 \mu \mathrm{sec}$ [2-4], three orders of magnitude longer than in III-V compounds [5], a consequence of the much smaller 
spin-orbit interaction (SOI) in Si [6]. Experimentally, the spin lifetimes have been determined both by standard electron-spin-resonance (ESR) experiments and by spin-echo experiments. The latter demonstrate directly a possible and very precise way of spin manipulation: in this method, spins are rotated by an angle of $\pi / 2$ or $\pi$, depending on the chosen microwave pulse length, and decoherence processes can be observed directly by looking at the echo amplitude [6].

In these experiments, a reasonable compromise between pulse amplitude and pulse width implies a pulse length of about 10 ns. The ratio of coherence time to manipulation time thus is only of the order of 100 whereas $10^{4}$ have been postulated [1]. We have shown earlier, however, that spin relaxation in Si quantum wells is governed by the Bychkov-Rashba effect [2] which results from one-sided modulation doping and the resulting loss in mirror symmetry. This effect has been shown to affect the linewidth (and thus the transverse spin relaxation time), the $g$-factor and the longitudinal spin relaxation time via the D'yakonov-Perel' mechanism [7]. We have shown that a spin coherence time of $25 \mu \mathrm{sec}$ can be expected for high mobility samples, if the asymmetry can be removed which leaves us with the intrinsic Elliott-Yafet spin relaxation [3, 8, 9]. For quantum dots made of III-V compounds the spin coherence time has been shown to increase still further so that it appears possible to fulfil the criterion of a ratio of $10^{4}$ for quantum dots in SiGe structures.

The other criterion mentioned is the possibility to select individual spin within an ensemble for manipulation. We proposed earlier to make use of the dependence of the $g$-factor on the alloy concentration in an $\mathrm{Si}_{1-x} \mathrm{Ge}_{x}$ alloy [4]. Applying an electric field perpendicular to the sample surface within a structure that exhibits a gradient of $x$ in growth direction $z$ will shift the wavefunction in $z$ direction [10] and as a consequence the $g$-factor can be "tuned" into or out of resonance when a microwave field of suitable frequency is applied. We have already demonstrated that the shift in resonance exceeds the linewidth already for $x>5 \%$. In this paper we investigate the effect of Ge in the channel in more detail on samples with a still higher mobility and we demonstrate that, like for pure Si channels, the Bychkov-Rashba field is also the most essential contribution for both spin relaxation and the g-factor. We evaluate the Bychkov-Rashba coefficient and we observe an increase in it with $x$ increasing from 0 to $10 \%$. This increase appears surprising at first glance since the barrier height decreases (the Ge concentration in the barrier $y$ remains almost constant in our sample series) and therefore we approach rather the case of bulk material with a field gradient. A possible conclusion from that will be that the higher SOI in Ge-alloyed $\mathrm{Si}$ or an additional $x$-gradient within the well is responsible for this effect.

\section{Experiment}

Samples were grown by molecular beam epitaxy on $3 \mu \mathrm{m}$ thick, step-graded and fully relaxed $\mathrm{Si}_{1-y} \mathrm{Ge}_{y}$ buffers (nominally $y_{\max }=0.25$ ) on top of a (100) $\mathrm{Si}$ 
substrate. The quantum well consists of $\mathrm{Si}_{1-x} \mathrm{Ge}_{x}(0 \leq x \leq 0.1)$, it has a thickness of $12 \mathrm{~nm}$ and thus it is pseudomorphic and strained. The strain causes a splitting of the $\Delta_{2}$ conduction band minima in such a way that only the ellipsoids with their main axis oriented perpendicular to the sample surface $\left(m^{*}=0.19 m_{0}\right)$ are populated. On top of this a spacer $\left(y_{\text {nom. }}=0.25, d=10 \mathrm{~nm}\right)$ and an Sb doped layer $\left(d=15 \mathrm{~nm},[\mathrm{Sb}]=10^{18} \mathrm{~cm}^{-3}\right.$ ) are grown, followed by another buffer (the same $y$ ) and a $10 \mathrm{~nm} \mathrm{Si} \mathrm{cap.}$

Although the Ge concentration is well reproducible within one run, it is difficult to predefine via the growth parameters in our MBE system. The experimental determination of $x$ and $y$ by X-ray techniques and secondary ion mass spectroscopy (SIMS) poses also some problems. Therefore we used low-energy Rutherford backscattering (500 keV He) to investigate the composition of our relatively complex layer sequence [11]. Results are given in the Table.

TABLE

Characteristics of investigated samples: channel Ge concentration $x$, barrier Ge concentration $y$, their difference, mobility $\mu$ of electrons at $2 \mathrm{~K}$ and concentration $n_{s}$ for three MBE grown, modulation doped $\mathrm{Si}_{1-x} \mathrm{Ge}_{x}$ quantum well samples.

\begin{tabular}{c|c|c|c|c}
\hline \hline$x$ (channel) & $y$ (barrier) & $(y-x)$ & $\mu\left(\mathrm{cm}^{2} / \mathrm{V} \mathrm{s}\right)$ & $n_{s}\left(\mathrm{~cm}^{-2}\right)$ \\
\hline $0.0 \%$ & $25 \%$ & $25 \%$ & 100000 & $6 \times 10^{11}$ \\
$4.0 \%$ & $19 \%$ & $15 \%$ & 25000 & $3 \times 10^{11}$ \\
$9.5 \%$ & $22 \%$ & $12.5 \%$ & 25000 & $3 \times 10^{11}$
\end{tabular}

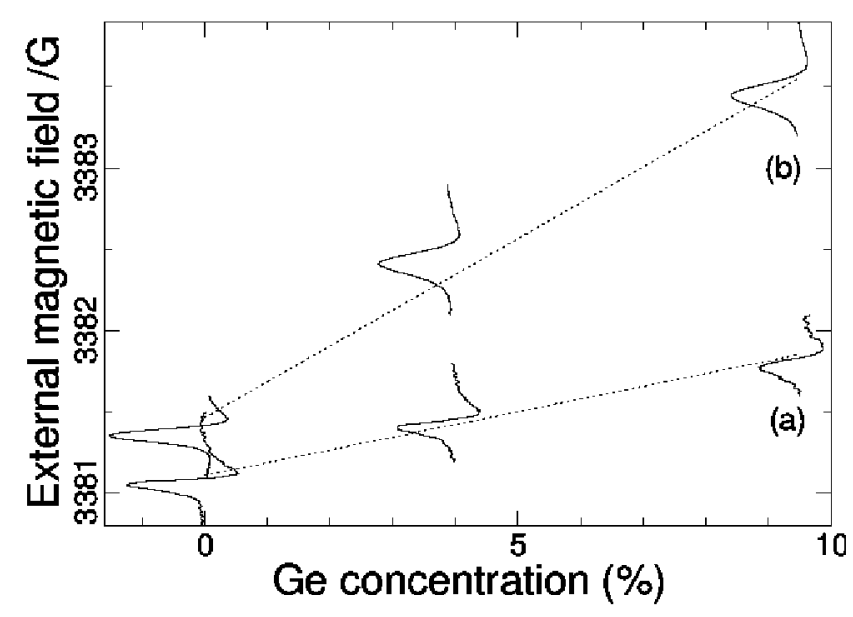

Fig. 1. ESR spectra measured at $2.5 \mathrm{~K}$ as a function of Ge concentration for (a) perpendicular, (b) in-plane magnetic field. 
We investigate the conduction electron spin resonance in modulation doped SiGe quantum wells in a standard X-band spectrometer (Bruker Elexsys E 580) with a TE102 cavity. Typical spectra are given in Fig. 1. In the upper half of the diagram, spectra are shown for perpendicular magnetic field, in the lower half - for in-plane field for the three samples with a nominal $x$ of $0,0.05$, and 0.1 , respectively. The spectra exhibit asymmetry approaching rather a Dysonian shape which has been explained in terms of electric dipole contributions [3]. It is clearly seen that the resonance field shifts towards higher field (lower $g$-factor) with increasing $x$ and at the same time the linewidth increases.

\section{Interpretation and discussion}

We have earlier shown in a detailed investigation of the influence of the electron density and the momentum relaxation time on the anisotropy of the $g$-factor and the ESR linewidth and also on the longitudinal spin relaxation of high mobility Si quantum wells that these quantities can be explained consistently in terms of the Bychkov-Rashba field, $\boldsymbol{H}_{\mathrm{BR}}$, which results from the one-sided modulation doping of our samples [3, 12]. In the present situation, the cyclotron frequency is in the range $\omega_{\mathrm{c}} \tau \sim 1$ and thus $\boldsymbol{H}_{\mathrm{BR}}$ can be treated as a small perturbation and we obtain for the $g$-factor [2]

$$
g=g_{0}\left[1+\frac{2 \pi n_{s} \alpha_{\mathrm{BR}}^{2}}{g_{0}^{2} \mu_{\mathrm{B}}^{2} H_{0}^{2}}\left(1+\cos ^{2} \theta\right)\right],
$$

where $\boldsymbol{H}_{0}$ is the static resonance field, $\theta$ - its inclination angle with respect to the surface normal, $g_{0}$ - the unperturbed $g$-factor of SiGe, $n_{s}$ - the 2D electron density and $\alpha_{\mathrm{BR}}$ stands for the Rashba coefficient which characterizes the SO interaction resulting from bi-linear terms in the $k$-vector and the spin in the Hamiltonian.

For the linewidth for in-plane field orientation we obtain $[2,13]$

$$
\Delta H=\frac{\pi \alpha_{\mathrm{BR}}^{2} n_{s} \tau}{2 \hbar g_{0} \mu_{\mathrm{B}}}
$$

Both quantities can be evaluated from the spectra shown in Fig. 1. The Rashba coefficient can be evaluated thus from the $g$-factor anisotropy and independently from the linewidth. Results are given in Fig. 2 as a function of alloy composition $x$.

From Fig. 1 we see that for $5 \%$ of Ge in the quantum well the shift in the resonance field exceeds the linewidth already by a factor of 5 for in-plane orientation and by roughly a factor 3 for perpendicular field. This shows that the resonances are clearly separated at an even lower Ge concentration. This is important because the spin coherence time will be longer for lower $x$. 


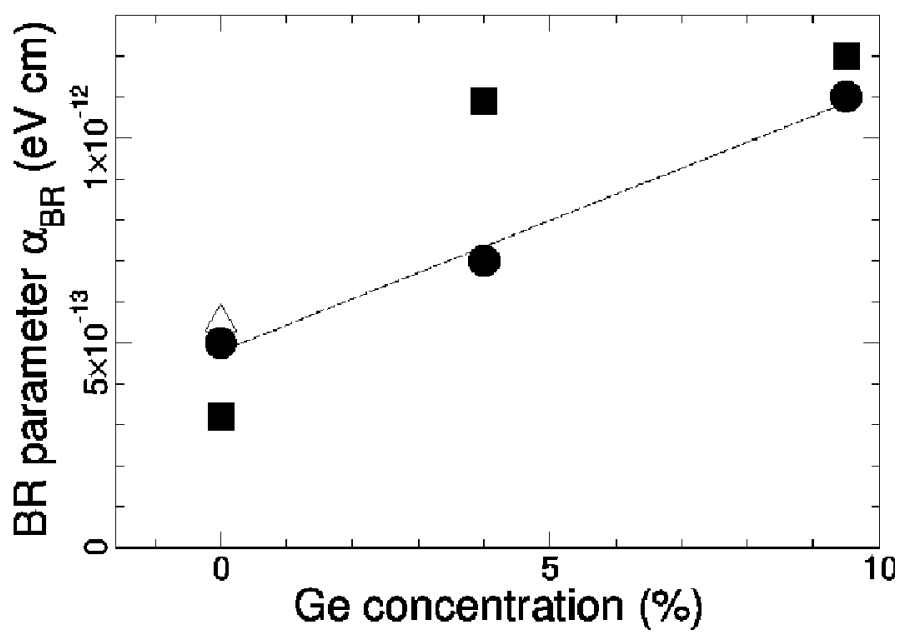

Fig. 2. BR parameter as a function of Ge concentration, calculated from $g$-factor anisotropy (solid circles) and from linewidth (solid squares). the triangle represents the BR parameter for a pure Si quantum well obtained earlier (see Ref. [2]).

We conclude thus that the use of different compositions of SiGe alloy can be a useful tool for the engineering of spintronic structures where it is important to have a possibility for selective spin manipulation by $g$-factor tuning.

Our evaluation of the BR field in SiGe QW shows that, in spite of the expectation based on the fact that that the height of the barrier decreases, the $\mathrm{BR}$ parameter in alloyed QW's is bigger than in a pure Si QW. This observation leads to the conclusion that either (i) the spin-obit parameter in the quantum well or (ii) a gradient of the Ge concentration within the QW can be an important source of the BR effect. The data show that this contribution can be even more important than an effective electric field in the QW and the asymmetry of the QW interfaces. Such a concept would also explain the puzzling effect observed in pure Si QW's where the BR parameter is almost independent of the electron concentration. The latter should screen the electric field and thus modify the penetration of the electron wave function into the barrier.

Work supported by the Fond zur Förderung der Wissenschaftlichen Forschung, Austria, OeAD and Gme, both Vienna, and in Poland under the grant PBZ-KBN 044/P03/2001.

\section{References}

[1] D.P. DiVincenzo, Fortschr. Phys. 48, 771 (2000).

[2] Z. Wilamowski, W. Jantsch, H. Malissa, U. Rössler, Phys. Rev. B 66, 195315 (2002).

[3] Z. Wilamowski, W. Jantsch, Phys. Rev. B 69, 035328 (2004). 
[4] W. Jantsch, Z. Wilamowski, N. Sandersfeld, M. Mühlberger, F. Schäffler, Physica E 13, 504 (2002).

[5] B. Beschoten, E. Johnston-Halperin, D.K. Young, M. Poggio, J.E. Grimaldi, S. Keller, S.P. DenBaars, U.K. Mishra, E.L. Hu, D.D. Awschalom, Phys. Rev. $B$ 63, 121202 (2001).

[6] Z. Wilamowski, W. Jantsch, N. Sandersfeld, M. Mühlberger, F. Schäffler, S. Lyon, Physica E 16, 111 (2003).

[7] M. D'yakonov, V. Perel', Sov. Phys. JETP 38, 177 (1973).

[8] R. Elliot, Phys. Rev. 96, 266 (1954).

[9] Y. Yafet, Solid State Phys. 14, 1 (1963).

[10] R. Vrijen, E. Yablonovitch, K. Wang, H.W. Jiang, A. Balandin, V. Roychowdhury, T. Mor, D.P. DiVincenzo, Phys. Rev. A 62, 012306 (2000).

[11] M. Draxler, M. Mühlberger, P. Bauer, in preparation.

[12] Y. Bychkov, E. Rashba, J. Phys. C 17, 6039 (1984).

[13] Z. Wilamowski, W. Jantsch, Physica E 12, 439 (2002). 